\title{
Indexing Medical Images Using the Wavelet Transform: Application to Retinal Images
}

\author{
${ }^{1}$ Souad Meziane Tani and ${ }^{2}$ Abdelhafid Bessaid \\ ${ }^{1}$ Department of Computer Science, Faculty of Science, \\ Laboratory of Biomedical Engineering (GBM), University Tlemcen 13000, Algeria \\ ${ }^{2}$ Department of Electrical and Electronics, Faculty of Technology, \\ Laboratory of Biomedical Engineering (GBM), University Tlemcen 13000, Algeria
}

Received 2014-05-07; Revised 2014-06-15; Accepted 2014-07-01

\begin{abstract}
Content-Based Image Retrieval Systems (CBIR) provide effective support to the doctor to find relevant images using their visual content. Current medical systems are unable to provide accurate and precise results. To solve this problem, effective indexing algorithms are required to extract the contents of the images. In this study, the method of indexing image from the energy coefficients of the wavelet transform is tested on the basis of retinal images to find similar images and the results are promising; the search precision is greater than $60 \%$.
\end{abstract}

Keywords: Indexing Image by Content, Wavelet Transform, Texture Analysis, CBIR

\section{INTRODUCTION}

The evolution of technology affected several sectors including medical sector where the appearance of devices that produce a large number of images each year. Rapid access tothese huge databases requires efficient indexing algorithms, Content Based Image Retrieval) are one of the possible solutions to efficiently manage this databases (Jai-Andaloussi et al., 2010).

The aim is to represent images using only their digital visual content. But what feature will best represent my digital visual content? The combination of all characteristics (shape, texture, color) gives the best description of the picture. However, it is rather complex to have a descriptor consisting of three heterogeneous characteristics, to reduce the complexity of the latter; we are interesting to global characteristics of the images, which do not require segmentation or extraction of particular features of the images such as form or contours. Thereby we focus on the analysis of texture using wavelet Transorm which has the advantage of providing a temporal and frequency information on the one hand and concentrate relevant information other, hence its use in image compression.

The setup of the article is as follows. Section II-A describes the database we used for evaluation. Section II-B describes the signature design process section II-C propose a distance between two signature; section III present results and section IV the conclusion and perspectives.

\section{MATERIALS AND METHODS}

\subsection{Database}

\subsubsection{Diabetic Retinopathy Database}

The database Diabetic Retinopathy (DR) database contains retinal images of diabetic patient, with associated anonymized information associated on the pathology. It is composed of 63 patient files containing 1045 photographs altogether (Lamard et al., 2007). Images have a definition of 1280 pixels/line for 1008 lines/image. They are lossless compressed Images. Patients were recruited in University Hospital of Brest since June 2003 and the images were

Corresponding Author: Souad Meziane Tani, Department of Computer Science, Faculty of Science,

$$
\text { Laboratory of Biomedical Engineering (GBM), University Tlemcen 13000, Algeria }
$$


acquired by experts using a Topcon Retinal digital Camera (TRC-50IA) connected to a computer. An example of image series is given in Fig. 1.

\subsection{Signature}

\subsubsection{The Wavelet Decomposition}

Wavelets are a family of functions generated from one single prototype function called mother wavelet $\psi$; by dilation and translation operations (ORACLE, 1989):

$$
\varphi(t)-\sqrt{2} \sum_{i=0}^{\infty} h(k) \varphi(2 t-k)
$$

where, $\mathrm{h}(\mathrm{k})$ are the scaling coefficients. Then, wavelet $\psi(t)$ is defined as (Nastar, 1997):

$$
\psi(t)-\sqrt{2} \sum_{k=\infty}^{\infty} g(k) \varphi(2 t-k)
$$

where, $g(k)=(-1)^{k} h(1-k)$ (Mallat, 1999), represent the wavelet coefficients.

The wavelet transform is the decomposition of a function into a family of wavelet functions $\psi_{m, \mathrm{n}}(\mathrm{t})$, where $\mathrm{m}$ is the index (scale/dilation) and $\mathrm{n}$ is the index (time/space). In other words using the wavelet transform, an arbitrary function may be written as a superposition of wavelets. Many constructions of wavelets have been mathematical (Combes et al., 1990) and signal processing literature (in the context of quadrature mirror filters) (Nguyen and Vaidyanathan, 1989). In the mid the introduction of multi-resolution analys wavelet transform by Mallat and Meyer has allowed the connection between the two approaches (Do and Vetterli, 2002). However, the wavelet transform can be seen as a filter bank a as follows onedimensional signal $\mathrm{x}[\mathrm{n}]$ :

- $\mathrm{X}[\mathrm{n}]$ is high pass and low pass filtered signals respectively $\mathrm{d}[\mathrm{n}]$ (detail) approximation)

- $\quad \mathrm{D}[\mathrm{n}]$ and $\mathrm{C}[\mathrm{n}]$ can be sub-sampled $2 \downarrow$ ) otherwise the transform is invariant wavelet (Fig. 2)

\subsection{Sub Band Characterization}

Several approaches have been proposed in the literature to characterize texture from the discrete wave lettrans form, the simplest and most popular approach for the construction of the signature from the wavelet coefficients is to form an energy vector of wavelet sub bands and the similarity measure is the Euclidean distance (Baaziz et al., 2010). The technique presented in
(Liu et al., 2008) extracts the signature from the coefficients of the wavelet packet transformed WPT of each sub-band. The 3 to WPT levels is applied to producing images of texture 64 subbands. Finally, the parameters of mean and variance are calculated for each sub-band to compose the feature vector of length 128 . Smeulders et al. (2000), multi-resolution analysis is used to create an index generated from the higher values (in absolute value) of the wavelet coefficients, it uses the Haar wavelet function and a suitable distance their representation. Nastar (1997), another technique based on the calculation of moments from the wavelet coefficients and the construction of histograms is proposed. Do and Vetterli (2002), the SM- KL statistical model is adopted and the Generalized Gaussian Distribution (GGD) proves a good fit to each sub-band of the Discrete Wavelet Transform (DWT). Signature is reduced to the estimation model parameters, two values per subband: Scale parameter and shape parameter. Assuming the independence of sub-bands the similarity measure is formulated as a combination of divergence between all sub-bands GGDs. In (Lamard et al., 2007) the authors use the generalized Gaussian density and the Kullback-Leibler divergence as similarity measure.

We have chosen to test the simplest approach to our diabetic retinopathy databases. Classical wavelets have been tested (Haar, daubechies 6 daubechies 2). We applied the wavelet transform on the green channel to the retinal image and the signature is composed of 10 energy $(3 n+1)$, each sub-band is characterized by energy. The Energy is given by Equation 1:

$$
\text { Energie }=\sum_{i}^{n} \sum_{j}^{n}\left(\operatorname{coef}(i, j)^{2}\right)
$$

where, coef $(i, j)$ represents the sub-band.

\subsection{Distance}

To compute the distance between two signatures, we calculate the Euclidean distance from each sub band (Fig. 3). The distance is a sum of the distance between corresponding sub bands of two images. The Euclidean Equation 2:

$$
\operatorname{Disr}(X, Y)=\sqrt{\sum_{i=1}^{n}|X i-Y i|^{2}}
$$

where, xi: Represents the (i) the image of the base and yi represents the (i) the query image. 

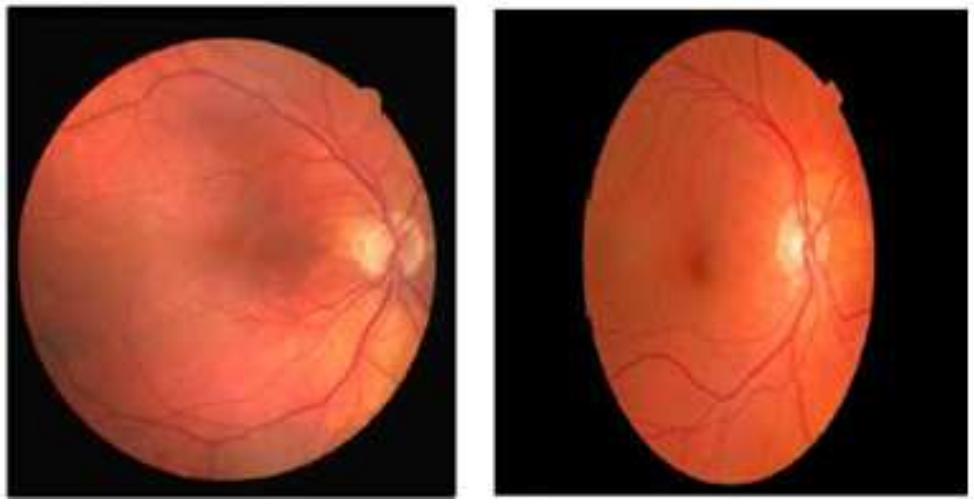

Fig 1. Photographs of patient eye

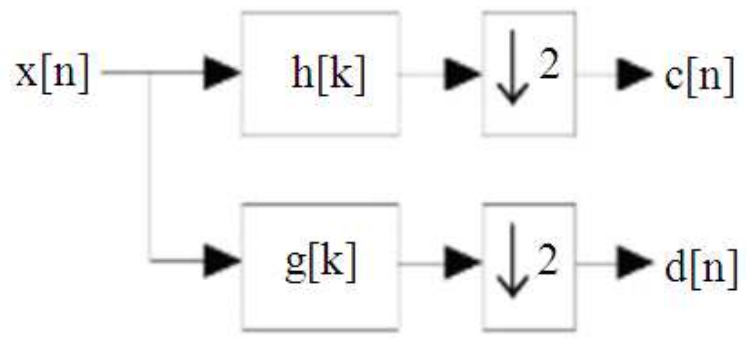

\section{RESULTS}

We tested the method based on energy to our Diabetic Retinopathy Database (RD). It contains various images i.e., a non-pathological image and pathological image corresponding to different stages of diabetic retinopathy. Each image in the database is used as a query and the algorithm find the five images of the database closet to the query. The evaluation criterion is the precision.

Precion: Is defined as the ratio of the number of

Fig. 2. Two-channel filter bank involving subsampled

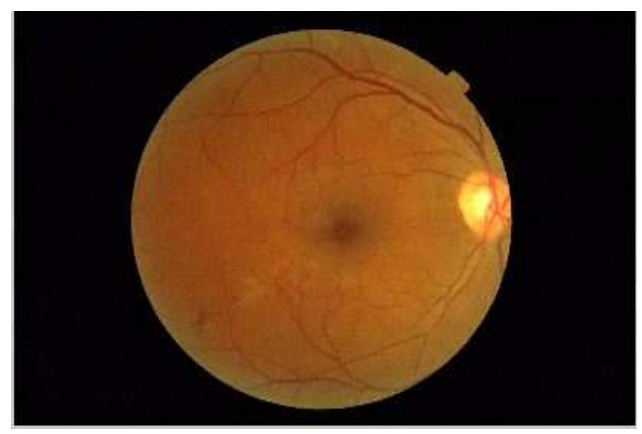
retrieved relevant images $r$ to the total number of retrieved images $\mathrm{n}$, i.e., $\mathrm{P}=\mathrm{r} / \mathrm{n}$ (Lakovidis et al., 2009), precison measures the accuracy of the retrieval. Precision is given by Equation 3:

Precision $=\frac{\text { number of relevant images retrieved }}{\text { Total number of images retrieved }} \times 100$

In Table 1, results are given of a 5 images window. The number 5 is the best compromise for physicians to perform a diagnostic. Results are compared with the previous CBIR system detailed in (Lamard et al., 2007).

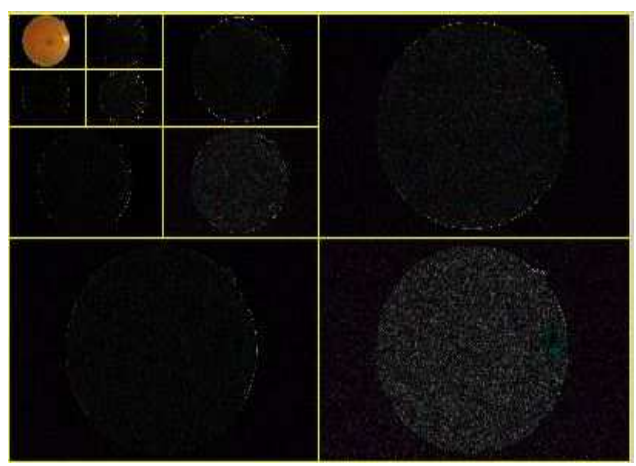
In Table 2, computing time for each approch is showns.

\section{DISCUSSION}

From Table 1, we note that the wavelet choice play an important role in the accuracy of the method and there are no suitable wavelet for all approaches presented in the table, however the energy-based approach gives a good result with Daubechies wavelet 6 .

We also compared the three approaches baths computing time which is very important for retrieval system based on content and the results shows that the energy-based approach gives a good accuracy and is fast enough.

Fig. 3. sub bands of two images 
Table 1. Means precision for 5 images window

\begin{tabular}{llll}
\hline $\begin{array}{l}\text { Wavelets } \\
\text { family }\end{array}$ & $\begin{array}{l}\text { Generalized } \\
\text { gaussians (\%) }\end{array}$ & $\begin{array}{l}\text { Energy } \\
\text { approach (\%) }\end{array}$ & $\begin{array}{c}\text { Histograms } \\
(\%)\end{array}$ \\
\hline Haar & 46,92 & 50,04 & 4240 \\
Daubechies 4 & 52,37 & 50,30 & 40,41 \\
Daubechies 6 & 52,13 & 60,04 & 51,4 \\
Daubechies9/7 & 50,05 & 53,60 & 49,9 \\
\hline
\end{tabular}

Table 2. Comparison of different approaches

\begin{tabular}{llll}
\hline Approachs & $\begin{array}{l}\text { Generalized } \\
\text { gaussians }\end{array}$ & $\begin{array}{l}\text { Energy } \\
\text { approach }\end{array}$ & Histograms \\
\hline Computing time $(\mathrm{sec})$ & $4.73 \mathrm{~s}$ & $0,44 \mathrm{~S}$ & $0.56 \mathrm{~s}$ \\
\hline
\end{tabular}

\section{CONCLUSION}

After testing approach based on the energy in our medical images (diabetic retinopathy databases), we conclude that the characterization of texture with the energy gives a result quite satisfy but remains insufficient and very discriminating view pathological image and another non-pathological may close while their content is very different, the same problem of the color histogram characteristic energies. However one based solely on the frequency content in the medical field descriptor is not sufficient, additional information that characterizes the semantic content of the image, for example the type of lesion present in the image, must be included in the descriptor of the image in order to enrich the signature and make the most discriminating and intelligent search system.

In baths of perspective is proposed to enrich and strengthen the descriptor with local information that characterizes lesions present in the image. i.e., characterizing the lesions present in the image to associate a semantic information descriptor that is the stage of diabetic retinopathy.

\section{REFERENCES}

Baaziz, N., O. Abahmane and R. Missaoui, 2010. Texture feature extraction in the spatial-frequency domain for content-based image retrieval. Cornell University Libraries.

Combes, J., A. Grossmann and P. Tchamitchian, 1990. Wavelets: Time-Frequency Methods and Phase Space. 2nd Edn., Springer-Verlag, ISBN-10: 0387511598, pp: 315

Do, M.N. and M. Vetterli, 2002. Wavelet-based texture retrieval using generalized Gaussian density and Kullback-Leibler distance. IEEE Trans. Image Proc., 11: 146-158. DOI: $10.1109 / 83.982822$
Jai-Andaloussi, S., M. Lamard, G. Gazuguel, H. Tairi and M. Meknassi et al., 2010. Content Based medical Image Retrieval based on BEMD: Optimization of a similarity metric. Proceedings of the Annual International Conference of the IEEE Engineering in Medicine and Biology Society, Aug. 31-Sept. 4, IEEE Xplore Press, Buenos Aires, pp: 3069-3072. DOI: 10.1109/IEMBS.2010.5626134

Lakovidis, D.K., N. Plekis, E.E. Katsifakos, I. Kopanakis and H. Karanikas, 2009. A pattern similarity sheme for medical image retrieval. IEEE Trans. Inform. Technol. Biomed., 13: 442-450. DOI: 10.1109/TITB.2008.923144

Lamard, M., G. Cazuguel, G. Quellec, L. Bekri and C. Roux et al., 2007. Content based image retrieval based on wavelet transform coefficients distribution. Proceeding of the 29th Annual International Conference of the IEEE Engineering in Medicine and Biology Society, Aug. 23-26, IEEE Xplore Press, Lyon, pp: 4532-4535. DOI: 10.1109/IEMBS.2007.4353347

Liu, M., Y. Hou, X. Zhu, D. Yang and X. Meng, 2008. New approach for texture classification based on concept. Proceeding of the Congress on Image and Signal Processing, May 27-30, IEEE Xplore Press, Sanya, China, pp: 160-164. DOI: 10.1109/CISP.2008.210

Mallat, S., 1999. Wavelet Tour of Signal Processing. 1st Edn., CBS Publishers and Distributors, ISBN-10: 8131202542. pp: 637.

Nastar, C., 1997. Indexation d'images par le contenu: Un etat de l'art.

Nguyen, T. and P. Vaidyanathan, 1989. Two-channel perfect-reconstruction fir qmf structures which yield linear-phase analysis and synthesis filters. IEEE Trans. Acoust. Speech Signal Proc., 37: 676-690. DOI: $10.1109 / 29.17560$

ORACLE, 1989. Oracle and sun microsystems. ORACLE.

Smeulders, A., M. Worring, S. Santini, A. Gupta and R. Jain, 2000. Content-based image retrieval at the end of the early years. IEEE Ransact. Patt. Anal. Mach. Intell., 22: 1349-1380. DOI: 10.1109/34.895972 